Gut and Liver, Vol. 11, No. 4, July 2017, pp. 489-496

\title{
Is the Reinitiation of Antiplatelet Agents Safe at 1 Week after Gastric Endoscopic Submucosal Dissection? Assessment of Bleeding Risk Using the Forrest Classification
}

\author{
Jong Yeul Lee, Chan Gyoo Kim, Soo-Jeong Cho, Young-II Kim, and II Ju Choi \\ Center for Gastric Cancer, National Cancer Center, Goyang, Korea
}

Background/Aims: Delayed bleeding after gastric endoscopic submucosal dissection (ESD) commonly occurs within 3 days, but it may also occur after 1 week following ESD, especially in antiplatelet agent users. We evaluated the risk of delayed bleeding in post-ESD ulcers using the Forrest classification. Methods: Registry data on the Forrest classification of post-ESD ulcers $(n=371)$ at 1 week or 2 weeks after ESD were retrospectively evaluated. The Forrest classification was categorized into two groups: increased risk (Forrest la to IIc) or low risk (Forrest III). The odds ratios (ORs) were calculated using logistic regression analysis. Results: Among 371 postESD ulcers, one ulcer (0.3\%) was classified as Forrest lb, two (0.5\%) as Forrest Ila, 17 (4.6\%) as Forrest Ilb, 172 (46.4\%) as Forrest Ilc, and 179 (48.2\%) as Forrest III. The proportion of increased-risk ulcers was $72.2 \%(140 / 194)$ at 1 week after ESD, which decreased to $29.4 \%$ (52/177) at 2 weeks after ESD $(p<0.001)$. In the multivariate analysis, a post-ESD ulcer at 1 week after ESD (OR, 7.54), younger age (OR, 2.17), and upper/middle ulcer location (OR, 2.05) were associated with increased-risk ulcers. Conclusions: One week after ESD, ulcers still have an increased risk of bleeding when assessed using the Forrest classification. This risk should be considered when resuming antiplatelet therapy. (Gut Liver 2017;11:489-496)

Key Words: Hemorrhage; Forrest classification; Stomach neoplasms; Endoscopic mucosal resection

\section{INTRODUCTION}

Endoscopic submucosal dissection (ESD) for gastric neoplasms has high en bloc and curative resection rates and low local recurrence rates, but it can cause more significant adverse events, such as perforation and bleeding, than endoscopic mucosal resection. ${ }^{1,2}$ Post-ESD bleeding occurs in approximately $4.1 \%$ to $15.3 \%$ of cases. ${ }^{2-7}$ Most post-ESD bleedings occur within 3 days, and the incidence markedly decreases during the first week. ${ }^{7,8}$ However, bleedings also occur after 1 week following ESD, especially in patients with risk factors, such as the use of antiplatelet agents. $^{5-7}$

According to the guidelines for endoscopic procedures in patients with antithrombotic treatment, patients with high thromboembolic risk are recommended to continue aspirin for the endoscopic procedures, even in high-risk procedures for bleeding, such as ESD. ${ }^{9-11}$ In our previous study, continuous aspirin use increased the bleeding risk, especially in those who resumed clopidogrel 7 days after ESD. ${ }^{5}$ Reinitiation of antithrombotic drug was also reported to increase the delayed bleeding risk, especially in patients using two or more antithrombotic drugs. ${ }^{6,12}$ However, other studies suggested that the continued use of aspirin only did not increase the bleeding risk after ESD. ${ }^{13,14}$ Therefore, the use of antithrombotic drugs and the bleeding risk after ESD remains controversial, and there is no consensus on the optimal timing for reinitiating antiplatelet therapy after ESD, especially in patients taking dual antiplatelet agents.

The Forrest classification was initially developed to describe upper gastrointestinal ulcers that were or had been bleeding. ${ }^{15}$ It is used to predict rebleeding rates and can stratify patients with acute upper gastrointestinal ulcer bleeding into high- and lowrisk categories for mortality. ${ }^{16,17}$ Therefore, this classification can be used to predict the delayed bleeding of post-ESD ulcers because it is simple to classify the post-ESD ulcer base, and because the risk of delayed bleeding can be estimated from this classification during surveillance endoscopy. ${ }^{18-20}$

In this study, we investigated the risk of delayed bleeding at post-ESD ulcers at 1 week or 2 weeks after the procedure using

Correspondence to: Il Ju Choi

Center for Gastric Cancer, National Cancer Center, 323 Ilsan-ro, Ilsandong-gu, Goyang 10408, Korea

Tel: +82-31-920-2282, Fax: +82-31-920-1127, E-mail: cij1224@ncc.re.kr

Received on May 6, 2016. Revised on August 19, 2016. Accepted on October 11, 2016. Published online March 24,2017

pISSN 1976-2283 eISSN 2005-1212 https://doi.org/10.5009/gnl16232

@ This is an Open Access article distributed under the terms of the Creative Commons Attribution Non-Commercial License (http://creativecommons.org/licenses/by-nc/4.0) which permits unrestricted non-commercial use, distribution, and reproduction in any medium, provided the original work is properly cited. 
the Forrest classification as a surrogate marker.

\section{MATERIALS AND METHODS}

\section{Patients}

This retrospective study was conducted using a prospectively collected database of consecutive patients at the National Cancer Center Hospital, Goyang, Korea. From August 2011 to June 2013, ESDs were performed to treat 573 gastric neoplasms, including early gastric cancer or adenoma, in 509 consecutive patients. Chromoendoscopies using indigo carmine and biopsies were done to determine whether ESD was indicated. During this period, the evaluations of post-ESD ulcers using Forrest classification were done at 1 week or 2 weeks after ESD for 405 post-ESD ulcers in 383 patients among 509 patient excluding 126 patients. Additional 13 ulcers in 12 patients were excluded because Forrest classification was impossible, and 21 smaller post-ESD ulcers in the same patient were also excluded. Thus, a total of 371 post-ESD ulcers in 371 patients were included in the analysis. A flow chart for inclusion in the analysis is shown in Fig. 1. Clinicopathologic features, comorbidities, the use of antiplatelet agents, and the Forrest classifications of post-ESD ulcers were obtained from the database. The Institutional Review Board of the National Cancer Center approved this study (NCC2015-0111).

\section{ESD technique}

ESD was performed by one of four expert endoscopists using the standard methods of our institution as previously described. ${ }^{21}$ Hemostatic procedures during ESD were performed as previously described. ${ }^{5}$ In brief, active bleeding during ESD was controlled using hemostatic forceps (Radial Jaw 3; Boston Scientific, Heredia, Costa Rica) with an electrosurgical generator
(VIO300D; ERBE Elektromedizin GmbH, Tübingen, Germany). At the end of the ESD procedure, preventive hemostasis was performed on oozing vessels or exposed vessels on the ulcer base. Ulcer diameters were estimated by measuring the maximal diameter of the resected specimens.

\section{Post-ESD management}

All patients received intravenous pantoprazole $(40 \mathrm{mg})$ twice daily for 2 days after ESD. Thereafter, they received oral pantoprazole (40 mg) twice daily. Hemoglobin levels were checked in the first post-ESD morning. If there was no adverse event, patients were allowed to have a liquid diet on the first day, and they were discharged on the second or third day. After discharge, patients took $40 \mathrm{mg}$ pantoprazole once a day for at least 28 days.

\section{Antiplatelet agents before and after ESD}

The strategy for using antiplatelet agents before and after ESD was made according to the patient's risk stratification based on the published criteria ${ }^{9,10}$ and our previously reported study results. ${ }^{5}$ A patient taking aspirin or other antiplatelet agents for the primary prevention of the thromboembolic disease without significant predisposing conditions was recommended to stop these medications 7 days before the procedure and to resume 4 weeks after ESD. For a patient with a high risk for thromboembolic disease taking dual antiplatelet agents, only aspirin was continued without cessation and concomitant antiplatelet agents were ceased at least 7 days before ESD and resumed 2 weeks after ESD. In patients using nonaspirin antiplatelet agents with a high risk for thromboembolic disease, replacement with aspirin was done 7 days before ESD and aspirin was continued as above.

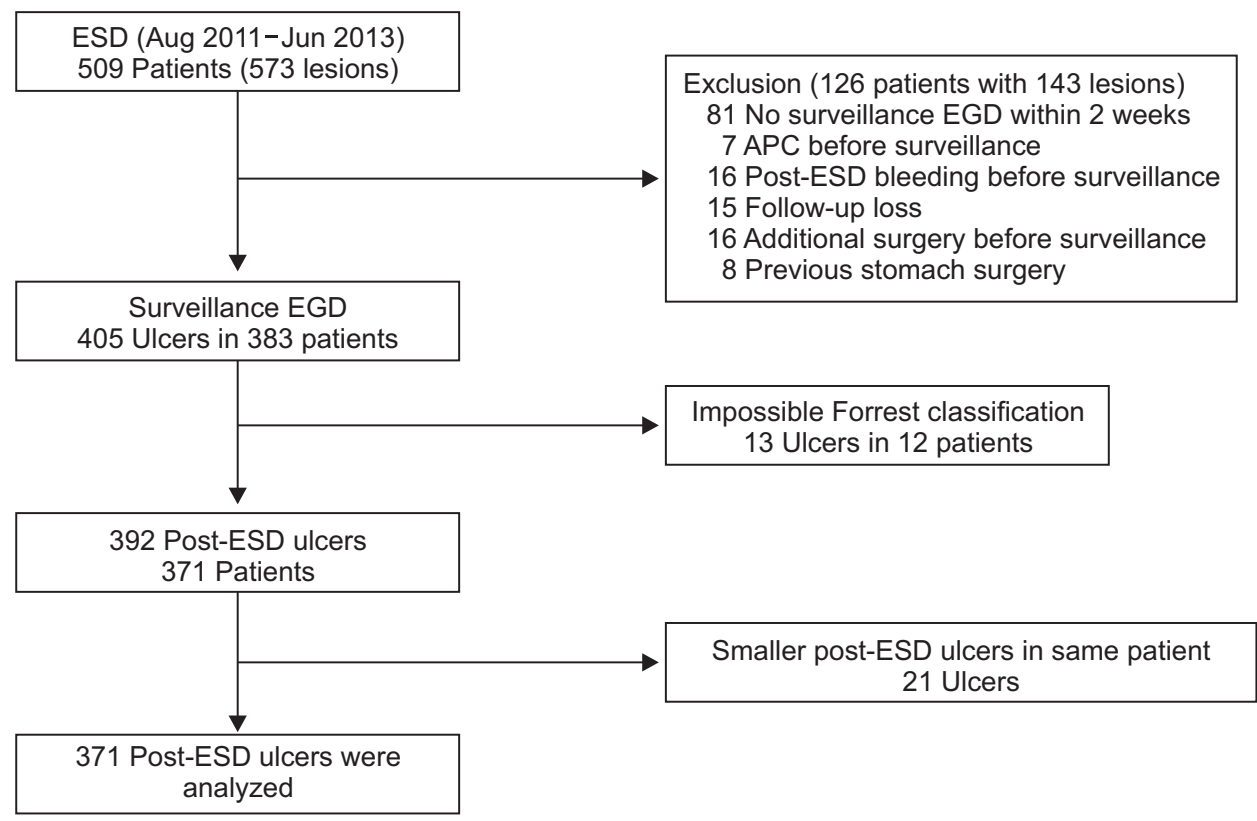

Fig. 1. Flow chart for inclusion in the analysis of the Forrest classification of post-ESD ulcers.

ESD, endoscopic submucosal dissection; APC, argon plasma coagulation. 


\section{Forrest classification of post-ESD ulcers}

During the first half of the study period (August 2011 to March 2012), esophagogastroduodenoscopy (EGD) was performed at 2 weeks after ESD, and during the latter half of the study period (April 2012 to June 2013) it was performed at 1 week after ESD. Surveillance EGD was done by the same endoscopist who performed ESD for the patient. The Forrest classification of post-ESD ulcers was determined as follows (Fig. 2): ulcers with spurting hemorrhage (Forrest Ia), oozing hemorrhage (Forrest Ib), visible vessel (Forrest IIa), adherent clot (Forrest IIb), flat pigmented spot on the base (Forrest IIc), and a clean ulcer base (Forrest III). ${ }^{15}$ Forrest classification was categorized into two groups using a simplified Forrest classification: $:^{22}$ increased risk group (Forrest Ia to IIc) or low risk group (Forrest III).

\section{Statistical analysis}

Continuous variables are presented as mean \pm standard deviation or as median with range. Statistical analysis was performed by the chi-square test for categorical variables and the Student t-test for continuous variables. Factors associated with the increased-risk ulcer group were assessed by logistic regression analysis using the estimated propensity scores for reducing bias. The propensity scores were estimated from the logistic regression model by including variables that could affect outcomes: sex, age, ulcer location, and ulcer size. After adjusting the estimated propensity scores, multivariate analysis was performed. Odds ratios (OR) with 95\% confidence intervals (CI) were calculated. Variables found to be significant factors by univariate analysis were included in the multivariate analysis. Two-sided p-values $<0.05$ were considered significant.

\section{RESULTS}

\section{Patient characteristics and clinicopathologic features of gastric neoplasms and post-ESD ulcers}

A total of 371 post-ESD ulcers in 371 patients were included in the analysis. Patient characteristics and clinicopathologic features of gastric neoplasms and post-ESD ulcers are summarized in Table 1. The mean age of the patients was 63.7 years, and $73.3 \%$ of the patients were male. Half of the patients had comorbid diseases (191/371, 51.5\%) and 17.5\% (65/371) were antiplatelet agent users. Among the antiplatelet agent users, 28 patients with a high risk for thromboembolic disease continued aspirin without cessation. Six patients with continued aspirin use were dual antiplatelet users, and they discontinued concomitant antiplatelet use 1 week before ESD and resumed 2 weeks after ESD. Patient characteristics and clinicopathologic features of post-ESD ulcers were not different between the 1 week and 2 week groups (Table 1).
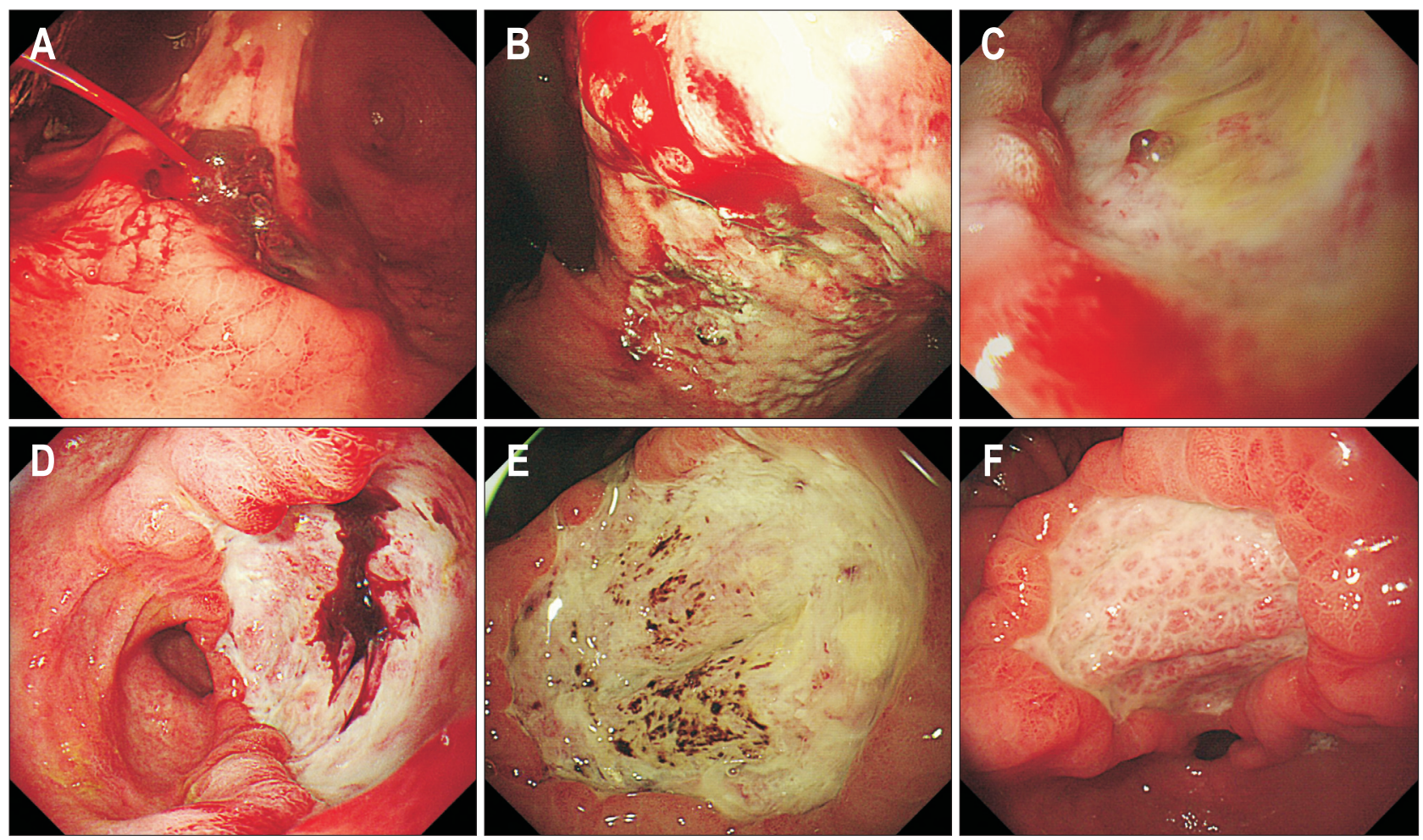

Fig. 2. Forrest classification of post-ESD ulcers. (A) Ulcers with a spurting hemorrhage (Forrest Ia). (B) Ulcers with an oozing hemorrhage (Forrest Ib). (C) Ulcers with visible vessels (Forrest IIa). (D) An adherent clot on the ulcer base (Forrest IIb). (E) Flat pigmented spot on the ulcer base (Forrest IIc). (F) A clean ulcer base (Forrest III).

ESD, endoscopic submucosal dissection. 
Table 1. Patient Characteristics and Clinicopathologic Features of Gastric Neoplasms and Post-ESD Ulcers

\begin{tabular}{|c|c|c|c|c|}
\hline \multirow[b]{2}{*}{ Characteristic } & \multicolumn{3}{|c|}{ All patient } & \multirow[b]{2}{*}{ p-value* } \\
\hline & $\begin{array}{c}\text { Total } \\
(\mathrm{n}=371)\end{array}$ & $\begin{array}{l}1 \text { Week after ESD } \\
\quad(\mathrm{n}=194)\end{array}$ & $\begin{array}{c}2 \text { Weeks after ESD } \\
(\mathrm{n}=177)\end{array}$ & \\
\hline \multicolumn{5}{|l|}{ Patients } \\
\hline \multicolumn{5}{|l|}{ Age, yr } \\
\hline $\mathrm{Mean}_{ \pm} \mathrm{SD}$ & $63.7 \pm 10.2$ & $64.4 \pm 10.1$ & $62.9 \pm 10.4$ & 0.159 \\
\hline Median (range) & $65.0(27-85)$ & $65.0(36-84)$ & $64.0(27-85)$ & \\
\hline$\leq 65$ & $197(53.1)$ & 99 (51.0) & $98(55.4)$ & 0.403 \\
\hline$>65$ & $174(46.9)$ & 95 (49.0) & 79 (44.6) & \\
\hline Male sex & $272(73.3)$ & $136(70.1)$ & $136(76.8)$ & 0.143 \\
\hline Comorbid condition & & & & 0.203 \\
\hline No & $180(48.5)$ & $88(45.4)$ & $92(52.0)$ & \\
\hline Yes & $191(51.5)$ & $106(54.6)$ & $85(48.0)$ & \\
\hline Hypertension & $153(41.2)$ & $89(45.9)$ & $64(36.2)$ & \\
\hline Diabetes mellitus & $67(18.1)$ & 38 (19.6) & $29(16.4)$ & \\
\hline Coronary artery disease & $17(4.6)$ & $11(5.7)$ & $6(3.4)$ & \\
\hline Atrial fibrillation & $5(1.3)$ & $3(1.5)$ & $2(1.1)$ & \\
\hline Valvular heart disease & $1(0.3)$ & 0 & $1(0.6)$ & \\
\hline Cerebrovascular disease & $15(4.0)$ & $8(4.1)$ & $7(4.0)$ & \\
\hline Peripheral vascular disease & $3(0.8)$ & $2(1.0)$ & $1(0.6)$ & \\
\hline Antiplatelet agents & & & & 0.539 \\
\hline None & $306(82.5)$ & $156(80.4)$ & $150(84.7)$ & \\
\hline Stopped & $37(10.0)$ & $22(11.3)$ & $15(8.5)$ & \\
\hline Continuous use & $28(7.5)$ & $16(8.2)$ & $12(6.8)$ & \\
\hline \multicolumn{5}{|l|}{ Tumor } \\
\hline Histology of main tumor & & & & 0.107 \\
\hline Adenoma & $67(18.1)$ & $41(21.1)$ & $26(14.7)$ & \\
\hline EGC & 304 (81.9) & 153 (78.9) & $151(85.3)$ & \\
\hline Tumor size, $\mathrm{cm}$ & & & & 0.556 \\
\hline $\mathrm{Mean}_{ \pm} \mathrm{SD}$ & $1.7 \pm 1.0$ & $1.8 \pm 1.0$ & $1.7 \pm 1.0$ & \\
\hline Median (range) & $1.5(0.2-5.2)$ & $1.5(0.3-5.2)$ & $1.5(0.2-5.0)$ & \\
\hline \multicolumn{5}{|l|}{ Post-ESD ulcer } \\
\hline Location within stomach & & & & 0.065 \\
\hline Lower & $288(77.6)$ & $158(81.4)$ & $130(73.4)$ & \\
\hline Upper/middle & $83(22.4)$ & 36 (18.6) & $47(26.6)$ & \\
\hline \multicolumn{5}{|l|}{ Ulcer size (long diameter), cm } \\
\hline $\mathrm{Mean}_{ \pm} \mathrm{SD}$ & $4.4 \pm 1.1$ & $4.4 \pm 1.2$ & $4.4 \pm 1.0$ & 0.934 \\
\hline$\leq 4.0$ & $180(48.5)$ & $90(46.4)$ & $90(50.8)$ & 0.391 \\
\hline$>4.0$ & $191(51.5)$ & $104(53.6)$ & $87(49.2)$ & \\
\hline
\end{tabular}

Data are presented as number (\%) unless otherwise indicated.

ESD, endoscopic submucosal dissection; SD, standard deviation; EGC, early gastric cancer.

*p-values for comparison between 1 week and 2 weeks after ESD.

\section{Forrest classifications of post-ESD ulcers and factors associated with increased-risk ulcers}

Table 2 shows the Forrest classifications of the post-ESD ulcers at 1 week or 2 weeks after ESD. When categorizing the For- rest classification into the increased-risk group (Forrest Ia to IIc) or the low-risk group (Forrest III), increased-risk ulcers (Forrest Ia to IIc) were more common at 1 week $(140 / 194,72.2 \%)$ than at 2 weeks $(52 / 177,29.4 \%)(p<0.001)$ after ESD. Forrest Ia to IIa ulcers were found in three cases at 1 week, but were not found 
Table 2. Forrest Classifications of Post-ESD Ulcers

\begin{tabular}{cccc}
\hline & \multicolumn{3}{c}{ All patient } \\
\cline { 2 - 3 } Forrest classification & $\begin{array}{c}\text { Total } \\
(\mathrm{n}=371)\end{array}$ & $\begin{array}{c}\text { Week after ESD } \\
(\mathrm{n}=194)\end{array}$ & $\begin{array}{c}2 \text { Weeks after ESD } \\
(\mathrm{n}=177)\end{array}$ \\
\hline Increased risk & $192(51.8)$ & $140(72.2)$ & $52(29.4)$ \\
Ib & $1(0.3)$ & $1(0.5)$ & 0 \\
IIa & $2(0.5)$ & $2(1.0)$ & 0 \\
IIb & $17(4.6)$ & $9(4.6)$ & $8(4.5)$ \\
IIc & $172(46.4)$ & $128(66.0)$ & $44(24.9)$ \\
Low risk & & & $125(70.6)$ \\
III & $179(48.2)$ & $54(27.8)$ & \\
\hline
\end{tabular}

Data are presented as number (\%).

ESD, endoscopic submucosal dissection.

*p-values were calculated between 1 week and 2 weeks after ESD according to the categorized risk groups (increased risk, Forrest Ia-IIc versus low risk, Forrest III).

Table 3. Factors Associated with Increased-Risk Ulcers* after ESD Using the Estimated Propensity Scores

\begin{tabular}{|c|c|c|c|c|}
\hline & \multicolumn{4}{|c|}{ Total $(n=371)$} \\
\hline & \multicolumn{2}{|c|}{ Univariate } & \multicolumn{2}{|c|}{ Multivariate } \\
\hline & OR $(95 \% \mathrm{CI})$ & p-value & OR $(95 \% \mathrm{CI})$ & p-value \\
\hline \multicolumn{5}{|l|}{ Sex } \\
\hline Male & 1 (Reference) & & & \\
\hline Female & $1.10(0.70-1.75)$ & 0.678 & - & - \\
\hline \multicolumn{5}{|l|}{ Age, yr } \\
\hline$>65$ & 1 (Reference) & & 1 (Reference) & \\
\hline$\leq 65$ & $1.55(1.03-2.33)$ & 0.037 & $2.17(1.29-3.64)$ & 0.003 \\
\hline \multicolumn{5}{|l|}{ Location } \\
\hline Lower & 1 (Reference) & & 1 (Reference) & \\
\hline Upper/middle & $1.46(0.89-2.40)$ & 0.133 & $2.05(1.02-4.09)$ & 0.043 \\
\hline \multicolumn{5}{|l|}{ Comorbid condition } \\
\hline No & 1 (Reference) & & & \\
\hline Yes & $1.20(0.80-1.80)$ & 0.388 & - & - \\
\hline \multicolumn{5}{|l|}{ Antiplatelet agents } \\
\hline None & 1 (Reference) & & 1 (Reference) & \\
\hline Stopped & $1.03(0.52-2.04)$ & 0.936 & $1.01(0.46-2.23)$ & 0.983 \\
\hline Continuous use & $1.75(0.78-3.92)$ & 0.171 & $2.15(0.84-5.48)$ & 0.110 \\
\hline \multicolumn{5}{|l|}{ Ulcer size, $\mathrm{cm}$} \\
\hline$\leq 4.0$ & 1 (Reference) & & 1 (Reference) & \\
\hline$>4.0$ & $1.49(0.99-2.24)$ & 0.057 & $1.49(0.91-2.43)$ & 0.109 \\
\hline \multicolumn{5}{|c|}{ Duration after ESD, wk } \\
\hline 2 & 1 (Reference) & & 1 (Reference) & \\
\hline 1 & $6.23(3.97-9.78)$ & $<0.001$ & $7.54(4.62-12.3)$ & $<0.001$ \\
\hline Propensity score & $1.09(0.12-10.3)$ & 0.937 & $1.35(0.05-39.8)$ & 0.862 \\
\hline
\end{tabular}

ESD, endoscopic submucosal dissection; OR, odds ratio; CI, confidence interval.

*Increased-risk ulcers were defined as Forrest Ia-IIc post-ESD ulcers. 
at 2 weeks after ESD.

Supplementary Tables 1 and 2 show the Forrest classifications of post ESD-ulcers according to use of antiplatelet agents (none, stopped, and continued) or ulcer location (lower and middle/ upper) at 1 week or 2 weeks. On subgroup analysis, there were no significant differences of the Forrest classification between antiplatelet agent users and nonusers at 1 week or at 2 weeks ( $\mathrm{p}=0.691$ and $\mathrm{p}=0.470$, respectively) (Supplementary Table 1). There was no significant difference of the Forrest classification between lower and middle/upper locations at 1 week $(p=0.993)$ (Supplementary Table 2). However, at 2 weeks, increased-risk ulcers were significantly higher in middle/upper location (48.9\%) than in lower location (22.3\%) $(\mathrm{p}=0.001)$.

Risk factors associated with the increased-risk group were assessed by logistic regression analysis (Table 3). Multivariate analyses determined that 1 week after ESD, the age $\leq 65$ years and middle/upper location of the ulcer in the stomach were significantly associated with increased-risk ulcers.

\section{DISCUSSION}

In the present study, we investigated the risk of delayed bleeding at post-ESD ulcers at 1 week or 2 weeks after ESD using the Forrest classification to determine the optimal timing for reinitiating antiplatelet therapy. We found that at 1 week, a high proportion of ESD-induced ulcers were increased-risk ulcers (Forrest Ia to IIc, 72.2\%), and the proportion decreased significantly at 2 weeks after the ESD when assessed by the Forrest classification. In addition, Forrest Ia to IIa lesions were found only in the 1 week group. Our results imply that at 1 week after the procedure, ESD-induced ulcers still have an increased risk of delayed bleeding.

Recent guidelines for gastroenterological endoscopy in patients with antithrombotic treatment recommend that patients with high thromboembolic risk continue aspirin, even if they are undergoing endoscopic procedures with a high risk for bleeding, such as ESD. ${ }^{9-11}$ Continued aspirin use during ESD is accepted, because most post-ESD bleedings can be successfully managed using endoscopic hemostasis, ${ }^{5,6,13}$ and discontinuation of antithrombotic drugs may result in thromboembolic events that cause severe morbidity or mortality. ${ }^{13,14,23}$ Some studies have shown that continued use of low-dose aspirin does not increase the risk of bleeding after ESD, ${ }^{13,14}$ while our previous study showed that post-ESD bleeding is higher in continuous aspirin users than in those with no or interrupted aspirin use. ${ }^{5}$ Furthermore, the resumption of clopidogrel at 1 week after ESD, combined with aspirin use, is significantly associated with delayed post-ESD bleeding (relative risk, 26.71). Additionally, Koh et al. ${ }^{6}$ showed that reinitiating antithrombotic drug therapy is a significant independent risk factor for delayed post-ESD bleeding. A recent multicenter prospective cohort study also reported that early resumption of a thienopyridine derivative is the only significant factor for post-ESD bleeding in patients with continuous aspirin use due to a high risk of thromboembolism. ${ }^{12}$ Therefore, we estimated the risk of bleeding at post-ESD ulcers using the Forrest classification to determine the optimal timing for the resumption of antiplatelet therapy in patients with high risk of thromboembolism.

In this study, we adopted the Forrest classification as a surrogate marker for estimating the risk of delayed bleeding at post-ESD ulcers because it is a simple method to classify postESD ulcer during endoscopy, and the risk of delayed bleeding is easily estimated from it. We categorized the original Forrest classification into a simplified Forrest classification ${ }^{22}$ with an increased-risk group (Forrest Ia to IIc) and a low-risk group (Forrest III). Our results showed that increased-risk ulcers were significantly higher at 1 week than at 2 weeks after ESD. Furthermore, no ulcers exceeding Forrest IIlb were found at 2 weeks after ESD. These results imply that ulcers still have a risk of delayed bleeding at 1 week after ESD, and this risk is significantly lower at 2 weeks after the procedure. Although the majority of post-ESD bleedings occurred within the first week, ${ }^{7,8}$ some occurred beyond 1 week after ESD, especially in patients taking antiplatelet agents. $^{5-7,12}$ Considering these reports and our results, reinitiating antiplatelet agents at 2 weeks after ESD may be safer than within 1 week, especially in patients taking dual antiplatelet agents with continuous aspirin use.

Although scheduled second-look endoscopy (SLE) was empirically performed after gastric ESD for the purpose of reducing post-ESD bleeding, recent randomized controlled trials for the efficacy of SLE concluded that SLE has little or no effect on the prevention of delayed bleeding. ${ }^{24-26}$ However, recent SLE studies using the Forrest classification reported that high-risk ulcer stigma (Forrest I to IIa) during SLE are significantly associated with delayed bleeding after SLE, although additional hemostasis had been carried out for active bleeding (Forrest I) or nonbleeding visible vessels (Forrest IIa) during SLE. ${ }^{18-20}$ These recent reports support our finding that ulcers still had a risk of bleeding at 1 week after ESD and our suggestion that resuming antiplatelet agents at 2 weeks after ESD might be safer than within 1 week. However, the Forrest classification distribution in previous SLE studies showed a higher proportion of active bleeding (Forrest $\mathrm{Ia} / \mathrm{Ib}$ ) or nonbleeding visible vessels (Forrest IIa) than did our study. ${ }^{18-20}$ This might be due to timing differences, because SLE was performed at 1 day post-ESD, which was earlier than in our study. ${ }^{18-20}$

Previous studies on risk factors for bleeding after ESD showed that the location of the lesion, larger resection specimen size, less operator experience, or lack of meticulous postESD coagulation of visible vessels is associated with increased bleeding risk. ${ }^{7,27,28}$ In our results, operator experience and postESD coagulation were not significant factors, because the four endoscopists who performed the ESDs had experienced at least 200 ESDs before this study, and meticulous preventive he- 
mostasis was routinely done at the end of the ESD procedure. Furthermore, ulcers located in the upper and middle portion of the stomach were significantly associated with increased risk in our study. However, the risk associated with the lesion location is inconsistent among studies. ${ }^{7,28,29}$ Takizawa et al. ${ }^{7}$ showed that the incidence of delayed bleeding in the middle and lower stomach is less with post-ESD coagulation. However, post-ESD coagulation is difficult for lesions located in the upper part of the stomach due to the difficulty of controlling and approaching the scope for preventive hemostasis. For these reasons, upper/middle ulcer location might have an association with the increased risk in our study.

In our study, younger patient age was also a significant factor for increased-risk ulcers. One prior study reported that the patient's age ( $\leq 65$ years; OR, 6.11) is a significant predictive variable for the delayed postendoscopic mucosal resection hemorrhage. ${ }^{30}$ They assumed that atrophic changes with age might reduce the vascular plexus in the stomach and affect the results. Another study found that older age (OR, 0.931; 95\% CI, 0.88 to 0.98 ) is a significant predictive factor for a successful ESD without bleeding, ${ }^{29}$ although age was not found to be a risk factor for bleeding after ESD in other studies. ${ }^{7,27,28,31}$ Further studies are needed to verify the association between the risk of delayed bleeding and different age groups.

A major strength of this study is that this is the first study to assess the Forrest classification of post-ESD ulcers at 1 week or 2 weeks after ESD. In addition, all patients were recommended to follow our institutional strategy for the use of antiplatelet agents before and after ESD. However, there were several limitations to this study. First, the timing of surveillance EGD for the Forrest classification was not randomly assigned. Instead, we performed EGD at 2 weeks after ESD consecutively during the first half of the study, and at 1 week during the latter half, which rendered no significant difference in baseline characteristics between the two groups. We also applied the propensity score adjustment to minimize the effects of biases on the clinical outcome. Second, the Forrest classification was originally developed for benign ulcers for predicting the risks for rebleeding and mortality. Post-ESD ulcers are artificial ulcers resulting from endoscopic resection and may have different pathophysiology and bleeding risks compared to those of benign peptic ulcers. Third, this study had a limited number of patients with continued use of aspirin (28/371, 7.5\%). Therefore, the association between continued use of aspirin and increased risk of ulcers or postESD bleeding may require further investigation.

In summary, our study showed that ESD-induced ulcers still had an increased risk of delayed bleeding at 1 week after ESD, and the risk decreased significantly at 2 weeks after ESD when assessed by the Forrest classification. Thus, this finding should be taken into consideration in clinical practice for gastric ESD patients that use antiplatelet agents. Further study is needed to determine the optimal time interval to reinitiate antiplatelet therapy, especially for dual antiplatelet agent users.

\section{CONFLICTS OF INTEREST}

No potential conflict of interest relevant to this article was reported.

\section{ACKNOWLEDGEMENTS}

This work was supported by Grant NCC-1610180 from the National Cancer Center, Korea.

Author contributions: conception and design of the study, I.J.C.; analysis and interpretation of the data, I.J.C. and J.Y.L.; drafting of the article, I.J.C. and J.Y.L.; critical revision of the article for important intellectual content, C.G.K., S.J.C., and Y.I.K.; statistical analysis, J.Y.L. and Y.I.K.; and final approval of the article, J.Y.L., I.J.C., C.G.K., S.J.C., and Y.I.K.

\section{REFERENCES}

1. Cao Y, Liao C, Tan A, Gao Y, Mo Z, Gao F. Meta-analysis of endoscopic submucosal dissection versus endoscopic mucosal resection for tumors of the gastrointestinal tract. Endoscopy 2009;41:751757.

2. Oka S, Tanaka S, Kaneko I, et al. Advantage of endoscopic submucosal dissection compared with EMR for early gastric cancer. Gastrointest Endosc 2006;64:877-883.

3. Goto O, Fujishiro M, Kodashima S, et al. A second-look endoscopy after endoscopic submucosal dissection for gastric epithelial neoplasm may be unnecessary: a retrospective analysis of postendoscopic submucosal dissection bleeding. Gastrointest Endosc 2010;71:241-248.

4. Saito I, Tsuji Y, Sakaguchi Y, et al. Complications related to gastric endoscopic submucosal dissection and their managements. Clin Endosc 2014;47:398-403.

5. Cho SJ, Choi IJ, Kim CG, et al. Aspirin use and bleeding risk after endoscopic submucosal dissection in patients with gastric neoplasms. Endoscopy 2012;44:114-121.

6. Koh R, Hirasawa K, Yahara S, et al. Antithrombotic drugs are risk factors for delayed postoperative bleeding after endoscopic submucosal dissection for gastric neoplasms. Gastrointest Endosc 2013;78:476-483.

7. Takizawa K, Oda I, Gotoda T, et al. Routine coagulation of visible vessels may prevent delayed bleeding after endoscopic submucosal dissection: an analysis of risk factors. Endoscopy 2008;40:179183.

8. Goto 0, Fujishiro M, Oda I, et al. A multicenter survey of the management after gastric endoscopic submucosal dissection related to postoperative bleeding. Dig Dis Sci 2012;57:435-439.

9. ASGE Standards of Practice Committee, Anderson MA, BenMenachem T, et al. Management of antithrombotic agents for endoscopic procedures. Gastrointest Endosc 2009;70:1060-1070. 
10. Boustière C, Veitch A, Vanbiervliet G, et al. Endoscopy and antiplatelet agents: European Society of Gastrointestinal Endoscopy (ESGE) guideline. Endoscopy 2011;43:445-461.

11. Fujimoto K, Fujishiro M, Kato M, et al. Guidelines for gastroenterological endoscopy in patients undergoing antithrombotic treatment. Dig Endosc 2014;26:1-14.

12. Ono S, Fujishiro M, Yoshida N, et al. Thienopyridine derivatives as risk factors for bleeding following high risk endoscopic treatments: Safe Treatment on Antiplatelets (STRAP) study. Endoscopy 2015;47:632-637.

13. Lim JH, Kim SG, Kim JW, et al. Do antiplatelets increase the risk of bleeding after endoscopic submucosal dissection of gastric neoplasms? Gastrointest Endosc 2012;75:719-727.

14. Sanomura Y, Oka S, Tanaka S, et al. Continued use of low-dose aspirin does not increase the risk of bleeding during or after endoscopic submucosal dissection for early gastric cancer. Gastric Cancer 2014;17:489-496.

15. Forrest JA, Finlayson ND, Shearman DJ. Endoscopy in gastrointestinal bleeding. Lancet 1974;2:394-397.

16. Laine L, Peterson WL. Bleeding peptic ulcer. N Engl J Med 1994;331:717-727.

17. Laine L, Jensen DM. Management of patients with ulcer bleeding. Am J Gastroenterol 2012;107:345-360.

18. Choi CW, Kim HW, Kang DH, et al. Clinical outcomes of secondlook endoscopy after gastric endoscopic submucosal dissection: predictive factors with high risks of bleeding. Surg Endosc 2014;28:2213-2220.

19. Noda H, Ogasawara N, Izawa S, et al. Risk factors for bleeding evaluated using the Forrest classification in Japanese patients after endoscopic submucosal dissection for early gastric neoplasm. Eur J Gastroenterol Hepatol 2015;27:1022-1029.

20. Ono S, Ono M, Nakagawa M, Shimizu Y, Kato M, Sakamoto N. Delayed bleeding and hemorrhage of mucosal defects after gastric endoscopic submucosal dissection on second-look endoscopy. Gastric Cancer 2016;19:561-567.

21. Lee JY, Choi IJ, Cho SJ, et al. Endoscopic submucosal dissection for metachronous tumor in the remnant stomach after distal gastrectomy. Surg Endosc 2010;24:1360-1366.
22. de Groot NL, van Oijen MG, Kessels K, et al. Reassessment of the predictive value of the Forrest classification for peptic ulcer rebleeding and mortality: can classification be simplified? Endoscopy 2014;46:46-52.

23. Fujishiro M, Oda I, Yamamoto Y, et al. Multi-center survey regarding the management of anticoagulation and antiplatelet therapy for endoscopic procedures in Japan. J Gastroenterol Hepatol 2009;24:214-218.

24. Ryu HY, Kim JW, Kim HS, et al. Second-look endoscopy is not associated with better clinical outcomes after gastric endoscopic submucosal dissection: a prospective, randomized, clinical trial analyzed on an as-treated basis. Gastrointest Endosc 2013;78:285294.

25. Kim JS, Chung MW, Chung CY, et al. The need for second-look endoscopy to prevent delayed bleeding after endoscopic submucosal dissection for gastric neoplasms: a prospective randomized trial. Gut Liver 2014;8:480-486.

26. Mochizuki S, Uedo N, Oda I, et al. Scheduled second-look endoscopy is not recommended after endoscopic submucosal dissection for gastric neoplasms (the SAFE trial): a multicentre prospective randomised controlled non-inferiority trial. Gut 2015;64:397-405.

27. Okada K, Yamamoto Y, Kasuga A, et al. Risk factors for delayed bleeding after endoscopic submucosal dissection for gastric neoplasm. Surg Endosc 2011;25:98-107.

28. Tsuji Y, Ohata K, Ito T, et al. Risk factors for bleeding after endoscopic submucosal dissection for gastric lesions. World J Gastroenterol 2010;16:2913-2917.

29. Jeon SW, Jung MK, Cho CM, et al. Predictors of immediate bleeding during endoscopic submucosal dissection in gastric lesions. Surg Endosc 2009;23:1974-1979.

30. Kim JW, Kim HS, Park DH, et al. Risk factors for delayed postendoscopic mucosal resection hemorrhage in patients with gastric tumor. Eur J Gastroenterol Hepatol 2007;19:409-415.

31. Jang JS, Choi SR, Graham DY, et al. Risk factors for immediate and delayed bleeding associated with endoscopic submucosal dissection of gastric neoplastic lesions. Scand J Gastroenterol 2009;44:1370-1376. 